\title{
Conhecimentos de acadêmicos da área da saúde sobre ventilação mecânica
}

\section{Knowledge of health academics about mechanical ventilation}

\author{
Paulo Ricardo de Sousa Vasconcelos' $\bullet$ Maria Aline Moreira Ximenes ${ }^{2} \bullet$ Ingrid Kelly Morais Oliveira ${ }^{3}$ \\ Maria Girlane Sousa Albuquerque Brandão ${ }^{4}$ Joselany Áfio Caetano ${ }^{5}$ Lívia Moreira Barros $^{6}$
}

\begin{abstract}
RESUMO
Objetivo:Avaliar o conhecimento teórico de acadêmicos sobre Ventilação Mecânica antes e após uma intervenção educativa. Método: Estudo documental, descritivo e retrospectivo com abordagem quantitativa, realizado durante julho de 2017 em um minicurso deVentilação Mecânica que avaliou o conhecimento dos participantes sobre a temática. Utilizou-se o teste binomial para verificar se a proporção de acertos entre os acadêmicos era superior a 70\% em cada questão ( $p<0,05)$. Resultados: Com uma amostra de 92 participantes, houve melhora do conhecimento em que se verificou aumento da frequência de acertos nas questões sobre Frequência Inspiratória (97,7\% - 43, p=0,999), Gasometria (84,1\% - 37, p=0,496), Equipe Multiprofissional (97,7\% - 43, $p=0,999)$, Modalidades Ventilatórias (97,7\% - 43, $p=0,999)$, Modo PSV (Ventilação com Pressão de Suporte) (86.4\% - 38, $p=0,663)$, Complicações do Ventilador Mecânico (88,6\% - 39, p=0,809) e Ciclagem por Pressão $(95,5 \%$ - 42, $\mathrm{p}=0$,993). Conclusão: Este estudo demonstrou uma mudança significativa no nível de conhecimento sobreVM dos participantes envolvidos evidenciando a eficácia da implementação de cursos de capacitação.

Palavras-chave: Respiração Artificial; Segurança do Paciente; Educação em Saúde.
\end{abstract}

\begin{abstract}
Objective:To evaluate the theoretical knowledge of students on Mechanical Ventilation before and after an educational intervention. Method: Documentary, descriptive and retrospective study with a quantitative approach, conducted during July 2017 in a mini-course on MechanicalVentilation that evaluated the participants' knowledge about the theme.The binomial test was used to verify whether the proportion of correct answers among students was greater than $70 \%$ in each question $(p<0.05)$. Results: With a sample of 92 participants, there was an improvement in knowledge in which there was an increase in the frequency of correct answers in questions about Inspiratory Frequency $(97.7 \%-43, p=0.999)$, Gasometry $(84.1 \%-37, p=$ 0.496), Multiprofessional Team (97.7\% - 43, $p=0.999)$,Ventilatory Modes (97.7\% - 43, $p=0.999)$, PSV Mode (Pressure Support Ventilation) $(86.4 \%-38, p=0.663)$, Complications of the Mechanical Ventilator $(88.6 \%-39, p=0.809)$ and Pressure Cycling $(95.5 \%-42, p=0.993)$. Conclusion:This study demonstrated a significant change in the level of knowledge about MV of the participants involved, showing the effectiveness of the implementation of training courses.

Keywords: Respiration Artificial; Patient Safety; Health Education.
\end{abstract}

NOTA

I Enfermeiro graduado pela Universidade EstadualVale do Acaraú (UVA).Departamento de Enfermagem. Sobral, Ceará, Brasil. E-mail: pauloricardo8923@gmail.com Orcid: https://orcid.org/0000-0002-9780-232X

2 Enfermeira. Mestranda em Enfermagem pela Universidade Federal do Ceará (UFC). Departamento de Enfermagem. Fortaleza, Ceará, Brasil. E-mail: aline.ximenesII@hotmail.com Orcid: https://orcid.org/0000-0002-I674-3357

3 Acadêmica de Enfermagem Universidade Estadual Vale do Acaraú (UVA). Departamento de Enfermagem. Sobral, Ceará, Brasil. E-mail: ingridkellyı.ik@gmail.com Orcid: https://orcid.org/0000-0003-1536-7289

4 Enfermeira. Mestranda em Enfermagem pela Universidade da Integração Internacional da Lusofonia Afro-Brasileira (UNILAB). Departamento de Enfermagem. Redenção, Ceará, Brasil. E-mail: girlane.albuquerque@yahoo.com.br Orcid: http://orcid.org/0000-0002-9925-4750

5 Doutora em Enfermagem. Docente da Universidade Federal do Ceará (UFC). Departamento de Enfermagem. Fortaleza, Ceará, Brasil. E-mail: joselany@ufc.br Orcid: http://orcid.org/0000-0002-0807-056X

6 Doutora em Enfermagem. Docente da Universidade da Integração Internacional da Lusofonia Afro-Brasileira (UNILAB). Departamento de Enfermagem. Redenção, CE, Brasil. E-mail: livia.moreirab@hotmail.com Orcid: http://orcid.org/0000-0002-0I74-2255 


\section{INTRODUÇÃO}

A assistência ventilatória permite a recuperação e manutenção do paciente com insuficiência cardiorrespiratória. Graças a essa intervenção muitas vidas de pacientes são salvas nas unidades de terapia intensiva (UTI). No entanto, o uso dessa terapia de suporte pode induzir danos estruturais irreversíveis e alterar a função respiratória e hemodinâmica causando complicações que impactam na evolução e no prognóstico dos pacientes ${ }^{(1)}$.

Estudo observacional realizado na Espanha com 3.588 adultos admitidos em Unidade de Terapia Intensiva identificou que 415 indivíduos necessitaram de ventilação mecânica prolongada, e destes 184 morreram por causas relacionadas à ventilação mecânica. Dos 3.173 pacientes que não necessitaram de aparelhos respiratórios houve um índice de mortalidade de apenas 3,1\%. Neste estudo a ventilação mecânica prolongada foi associada a mais complicações ${ }^{(2)}$. Em São Paulo, pesquisa realizada durante seis anos em uma unidade de terapia intensiva evidenciou uma taxa de mortalidade de $42,5 \%$ dos pacientes submetidos à ventilação mecânica, sendo a principal causa de morte as Infecções do Trato Respiratório (IRAS) ${ }^{(3)}$.

Refletindo sobre a elevada taxa de mortalidade associada à ventilação mecânica e também a quantidade de pacientes intubados e traqueostomizados internados em ambientes de cuidados intensivos, considera-se pertinente o aprimoramento do conhecimento acadêmico e profissional específicos sobre VM com a finalidade de proporcionar cuidados em conformidade com a cultura de segurança do paciente associado à monitorização dos parâmetros ventilatórios e reconhecimento de possíveis complicações ${ }^{(4)}$.

Uma assistência adequada é fator determinante no cuidado ao doente crítico em VM, principalmente na manutenção da oxigenação e perfusão suficiente para os órgãos e tecidos a fim de prevenir a hipóxia. Também cabe os profissionais reduzir as complicações, garantindo a sincronização da respiração do paciente como ventilador e o bom funcionamento do ventilador mecânico(5).

Os profissionais e acadêmicos, principalmente de enfermagem, por manterem contato direto e quase ininterrupto com os pacientes, desempenham importante papel no desenvolvimento e implementação de programas de prevenção de IRAS. Outros profissionais e estudantes da equipe, como os fisioterapeutas, médicos e nutricionistas, também podem contribuir para prevenção desse evento adverso. Contudo, para que medidas eficazes sejam adotadas, é primordial que esses profissionais juntamente com acadêmicos tenham conhecimentos específicos relacionados aos cuidados de prevenção.

Segundo a Anvisa (2017) umas das formas de reduzir os agravos relacionados VM é por meio de treinamentos ou educação continuada, inserindo novas estratégias educacionais de acordo com práticas baseadas em evidências e que se adaptem às necessidades de aprendizagem do público e das instituições ${ }^{(6)}$.

Logo, a identificação do nível de compreensão dos acadêmicos sobre VM torna-se relevante, pois permite aferir tanto o conhecimento acerca da temática quanto a presença de possíveis lacunas que poderão ser contornadas em espaços educativos, contribuindo assim para o fortalecimento de ações de manuseio e de prevenção das complicações causadas pelo uso do VM, implementando ações que podem resolver a angústia respiratória aguda, dispneia e o aumento do trabalho respiratório e prevenir eventos adversos.

Dessa forma, este estudo objetivou avaliar o conhecimento teórico de acadêmicos sobre Ventilação Mecânica antes e após uma intervenção educativa.

\section{MÉTODO}

Estudo documental, descritivo e retrospectivo com abordagem quantitativa. Os dados deste estudo foram provenientes de um minicurso de Ventilação Mecânica ofertado na Jornada Cearense de Urgência e Emergência na cidade de Sobral-CE durante julho de 2017. Nessa jornada, participaram 235 profissionais e acadêmicos da área da saúde e foram realizados sete minicursos: $\mathrm{Nu}$ trição do Paciente Crítico; Imobilizações; Ressuscitação Cardiopulmonar;Ventilação Mecânica;Avaliação Primária; Manobras de resgate.

O minicurso de Ventilação Mecânica teve a participação de 99 pessoas e, durante a sua realização, foram aplicados testes de conhecimento antes e após a aula teórica. Dentre os participantes havia acadêmicos de medicina, de enfermagem, de fisioterapia e de nutrição. Assim, a população-alvo do estudo foi representada pelos testes de conhecimento respondidos nesse momento. Foram incluídos na amostra os instrumentos de participantes que responderam pré e pós-teste e que tivesse $80 \%$ das informações preenchidas, sendo excluídos os instrumentos de participantes que não responderam o pós-teste diante da impossibilidade de comparação dos resultados antes e depois da intervenção educativa, totalizando uma amostra de 92 participantes.

O minicurso teve duração de $8 \mathrm{~h}$ e o método de ensino escolhido foi a aula expositiva dialogada com o uso de slides em Power Point com temas relacionados aos parâmetros de Ventilação Mecânica, Modos Ventilatórios, Pressão Positiva Expiratória Final,Técnica de Desmame, entre outros. A coleta de dados foi realizada a partir de um espelho do teste de conhecimento, o qual foi elaborado pelo pesquisador com base na literatura científica. $O$ instrumento continha 16 questões sobre suporte ventilatório, gasometria, modo de operação do ventilador mecânico, técnica de desmame, parâmetros, entre outros. 
O instrumento foi aplicado em auditório reservado para o minicurso deVM antes do início das atividades teóricas no período da manhã, sendo reaplicado ao fim do dia no período da tarde. Os participantes foram esclarecidos sobre o objetivo da aplicação do teste de conhecimento, sendo colhida a assinatura do termo de consentimento livre e esclarecido. Foi delimitado 20 minutos para resolução da atividade.

Após a coleta, os dados foram analisados estatisticamente por meio do programa Microsoft Excel 2007 e submetidos a uma análise descritiva por meio de percentuais e uma análise inferencial através do software $R$ com o teste binomial, com nível de significância de $5 \%$ $(p>0,05)$ para rejeitar a hipótese nula e indicar que os participantes tiveram uma concordância de acertos de cada questão igual ou superior a $70 \%$.

A coleta de dados ocorreu após a aprovação do Comitê de Ética e Pesquisa, sob parecer consubstanciado de número 2.578 .33 I com CAAE de número $860434 \mid 8.1 .0000 .5053$, de forma a garantir a proteção do sujeito do estudo em todas as fases da pesquisa, garantindo, portanto, o sigilo, levando em consideração a Resolução 466/12 do Conselho Nacional de Saúde, que trata da pesquisa envolvendo seres humanos, e traz como princípios a beneficência, a não maleficência, autonomia, justiça e equidade.

\section{RESULTADOS}

Na tabela I, apresenta-se a comparação de acertos sobre ventilação mecânica antes e após a participação no minicurso.

É possível observar que os participantes tiveram percentual de acertos superior a $70 \%$ no pré-teste apenas nas questões relacionadas aos Métodos Invasivos e Não Invasivos (93,2\% - 4I, $p=0,97)$, Frequência Inspiratória $(77,3 \%-34, p=0.113)$, Equipe Multiprofissional $(77,3 \%$ - 34, $p=0.113)$, Modalidades Ventilatórias $(84,1 \%-37$, $\mathrm{p}=0.496)$, Parâmetros Ventilatórios $(84,1 \%-37, \mathrm{p}=0.496)$ e Complicações do Ventilador Mecânico (81,8\% - 36, $\mathrm{p}=0.336)^{(\text {(Tabela I). }}$.

Já no pós-teste, houve melhora do conhecimento entre os participantes em que se verificou aumento da frequência de acertos nas questões sobre Frequência Inspiratória $(97,7 \%-43, p=0.999)$, Gasometria (84,I\% - 37, $p=0.496)$, Modo de Operação Combinada (SIMV) $(81,8 \%-36, p=0.336)$, Equipe Multiprofissional $(97,7 \%-43, p=0.999)$, Modalidades Ventilatórias $(97,7 \%$ - 43, $p=0.999$ ), Pressão Positiva Expiratória Final (PEEP) (77,3\% - 34, $p=0.113)$, Parâmetros Ventilatórios (70,5\% - 3I, p=0.010), Modo PSV (Ventilação com Pressão de Suporte) $(86.4 \%-38, p=0.663)$, Complicações do Ventilador Mecânico (88,6\% - 39, $p=0.809)$, Ciclagem por Pressão $(95,5 \%-42, p=0.993)$ e Pausa Inspiratória $(79,5 \%$ - 35, $p=0.205$ ) (Tabela I).

\section{DISCUSSÃO}

Os resultados obtidos na presente investigação

TABELA 1 - Comparação do total de acertos antes e após estratégia educativa. Sobral, CE, Brasil, 2018.

\begin{tabular}{|c|c|c|c|c|c|c|}
\hline \multirow{2}{*}{ Questão } & \multicolumn{2}{|c|}{ Acertos pré-teste* } & \multirow{2}{*}{ p-valort } & \multicolumn{2}{|c|}{ Acertos pós-teste* } & \multirow{2}{*}{ p-valort } \\
\hline & $\mathrm{N}$ & $\%$ & & $\mathrm{~N}$ & $\%$ & \\
\hline 1. Métodos Invasivos e Não Invasivos & 41 & 93,2 & 0.97 & 29 & 65,9 & 0.001 \\
\hline 2. Frequência Inspiratória & 34 & 77,3 & 0.113 & 43 & 97,7 & 0.999 \\
\hline 3. Objetivo do Ventilador Mecânico & 12 & 27,3 & $<0,000$ & 25 & 56,8 & $<0,000$ \\
\hline 4. Gasometria & 28 & 63,6 & 0.000 & 37 & 84,1 & 0.496 \\
\hline 5. Modo de Operação Combinada (SIMV) & 15 & 34,1 & $<0,000$ & 36 & 81,8 & 0.336 \\
\hline 6. Equipe Multiprofissional & 34 & 77,3 & 0.113 & 43 & 97,7 & 0.999 \\
\hline 7. Modalidades Ventilatórias & 37 & 84,1 & 0.496 & 43 & 97,7 & 0.999 \\
\hline $\begin{array}{l}\text { 8. Modalidade Assistida Controlado a Pressão } \\
\text { (PCV A/C) }\end{array}$ & 11 & 25,0 & $<0,000$ & 12 & 27,3 & $<0,000$ \\
\hline 9. Pressão Positiva Expiratória Final (PEEP) & 30 & 68,2 & 0.003 & 34 & 77,3 & 0.113 \\
\hline 10. Parâmetros Ventilatórios & 37 & 84,1 & 0.496 & 31 & 70,5 & 0.010 \\
\hline 11. Modo PSV (Ventilação com Pressão de Suporte) & 21 & 47,7 & $<0,000$ & 38 & 86,4 & 0.663 \\
\hline 12. Desmame do TOT & 21 & 47,7 & $<0,000$ & 21 & 47,7 & $<0,000$ \\
\hline 13. Complicações do Ventilador Mecânico & 36 & 81,8 & 0.336 & 39 & 88,6 & 0.809 \\
\hline 14. Ciclagem por Pressão & 27 & 61,4 & 0.000 & 42 & 95,5 & 0.993 \\
\hline 15. Pausa Inspiratória & 24 & 54,5 & $<0,000$ & 35 & 79,5 & 0.205 \\
\hline 16. Músculos Inspiratórios & 8 & 18,2 & $<0,000$ & 10 & 22,7 & $<0,000$ \\
\hline
\end{tabular}

*Frequência e porcentagem de acertos do total de estudantes avaliados; †Teste Binomial. 
apontaram percentuais razoáveis de conhecimento dos acadêmicos antes da intervenção educativa, os quais apresentaram aumento significativo após a ação educativa. A ventilação mecânica é, sem dúvida, um tema importante a ser considerado no processo de formação de profissionais da área da saúde, pois envolve aspectos diversos referentes ao perfil de pacientes e ao aparato tecnológico, necessitando da atuação da equipe multiprofissional. É de suma importância que os acadêmicos da área saúde estejam inseridos com competência e habilidades nesse contexto para prestar uma assistência segura ao paciente crítico.

Estudo realizado em Londrina evidenciou os benefícios de ações educativas com enfermeiros sobre prevenção de infecções relacionadas à ventilação mecânica, posto que houve adesão as práticas recomendadas ${ }^{(7)}$. Nos Estados Unidos um estudo demonstrou que um conjunto de intervenções educativas com residentes foi eficaz em aumentar a competência, o conhecimento e a confiança com o gerenciamento do ventilador mecânico durante situações de emergência ${ }^{(8)}$.

$\mathrm{Na}$ questão sobre Métodos Invasivos e Não Invasivos para Suporte ventilatório, observou-se que houve uma diminuição do número de acertos entre pré e pós-teste, o que pode estar relacionado ao pouco enfoque sobre os métodos de oxigenoterapia disponíveis durante o minicurso. É importante que o enfermeiro tenha conhecimento referente à aplicação de um suporte ventilatório sem recorrer a métodos invasivos e saiba identificar os critérios de falência desta modalidade ventilatória e a necessidade de substituir por VM invasiva. Uma vez que a evidência científica é soberana para tomada de decisões imediatas pensando na qualidade da assistência que não levará prejuízos aos pacientes críticos ${ }^{(4,9)}$. Portanto, é importante que os acadêmicos tenham conhecimentos sobre o objetivo da oxigenoterapia e da VM para que essas terapêuticas sejam implantadas somente quando o paciente tiver necessidade.

Em relação à questão que abordava sobre o objetivo do uso do Ventilador Mecânico, foi possível verificar um aumento de cerca de $30 \%$ de acertos entre o pré e pósteste. Corroborando com os resultados desse estudo, uma pesquisa realizada durante dois anos na Finlândia sobre uma intervenção educativa com uso de simulação sobre ventilação mecânica, evidenciou que a pós a intervenção, a pontuação média de conhecimentos aumentou de $46,8 \%$ para $58,8 \%$ da pontuação total na medição final pós-intervenção( ${ }^{(10)}$.

$\mathrm{Na}$ questão sobre gasometria, também houve aumento dos acertos entre os participantes, o qual foi estatisticamente significante, demonstrando que a intervenção educativa foi eficaz no ensino sobre a importância da realização da gasometria em pacientes em uso de VM. A capacidade de colher e analisar a gasometria arterial, relacionando-a a clínica do paciente, é uma função importante do profissional de saúde, pois fornece uma visão geral da situação do paciente em relação a sua oxigenação, ventilação e estado ácido-básico(II).

Estudo realizado na Índia buscou avaliar o nível de conhecimento de 30 profissionais enfermeiros sobre interpretação de gasometria arterial, foi evidenciado que 12 enfermeiros atingiram uma pontuação maior que $65 \%$ e os demais apresentaram acertos entre 13 a $40 \%$. Foi observado ainda que os maiores acertos estiveram relacionados a formação e tempo de experiência em UTI(12).

A enfermagem, em particular, deve se apropriar de procedimentos de sua competência, como é o caso da gasometria arterial, de forma que profissionais e acadêmicos inseridos no contexto prático possam fazê-la sem dificuldade de forma correta e segura para o paciente.

Quando questionados sobre o uso do modo Ventilação Mandatória Intermitente como a mais indicada na UTI por estar associada à diminuição do tempo de retirada do ventilador mecânico, verificou-se que, no pós-teste, o número de acertos dobrou comparado ao pré-teste, corroborando com os resultados obtidos em estudo realizado na UTI de um hospital público de médio porte localizado no interior do Ceará com uma amostra de 70 pacientes, que quanto a modalidade ventilatória, sobressaiu-se a modalidade Assistido/Controlada (A/C) identificada em $84,3 \%$ dos casos, seguida da Ventilação por Pressão de Suporte (PSV) com 8,6\% e a Ventilação Mandatória Intermitente com $7,1 \%{ }^{(13)}$. Isso demonstra o quanto foi relevante a intervenção educativa para o conhecimento dos participantes, pois saber qual a modalidade de maior eficácia é imprescindível para a sobrevida de um paciente.

No que se refere às complicações associadas à $\mathrm{VM}$, observou-se que, tanto no pré-teste como no pós-teste, os participantes tiveram uma frequência de acertos superior a $80 \%$. Este resultado torna-se relevante, pois o profissional deve estar ciente de todas as ações que ele pode realizar caso o paciente apresente alguma alteração. Tendo conhecimento específico e realizando esses cuidados diariamente a probabilidade de perceber alterações na saúde do paciente é muito maior e as ações tomadas imediatamente podem evitar sequelas e complicações irreversíveis ${ }^{(4)}$. Um estudo multicêntrico de coorte que envolveu todos os pacientes admitidos em quatro UTIs, identificou que pacientes com necessidade de VM tiveram um risco maior de complicações durante a permanência na UTI, taxas mais elevadas de mortalidade e custos maiores relativos à doença ${ }^{(14)}$. Portanto, quanto mais capacitado o profissional for para atuar em situações dessa natureza mais chances os pacientes terão de sobreviver. 
Verificou-se ainda que, apesar da implementação da intervenção educativa, os participantes apresentaram acertos insatisfatórios tanto no pré como no pós-teste sobre Modalidade Assistida Controlado a Pressão (PCV $\mathrm{A} / \mathrm{C}$ ) e Treinamento de Músculos Inspiratórios (TMI). Entretanto, quando questionados sobre Pressão Positiva Expiratória Final (PEEP), foi possível observar conhecimento satisfatório relacionado a esse aspecto, destacando a importância de os acadêmicos terem domínio sobre o assunto para não favorecer a ocorrência de eventos adversos relacionados à via aérea.

Na questão sobre o uso de $\mathrm{FiO} \square$ a $100 \%$ no início da VM para evitar hipoxemia arterial, foi observado redução no número de acertos no pós-teste, indicando a necessidade de reforço teórico sobre a temática. Ao contrário, na questão referente ao modo PSV, foi observado um aumento significativo de acertos. De acordo com as Diretrizes Brasileiras de Ventilação Mecânica ${ }^{(15)}$, a Pressão de Suporte (PSV) é considerado o modo preferencial durante a ventilação assistida ou espontânea, sendo que o seu uso deve ser iniciado o mais precocemente possível, conforme o quadro clínico.

Outra questão que teve frequência de acertos estatisticamente significantes no pós-teste foi sobre a correlação entre a ciclagem por pressão, o volume corrente e o tempo inspiratório associadas à complacência do aparelho. Neste mesmo estudo ${ }^{(13)}$, os mecanismos de ciclagem mais utilizados nos ajustes do ventilador foram à ciclagem a volume em $72,9 \%$ dos casos e a ciclagem a pressão em $27,1 \%$ dos pacientes. Sendo que o conhecimento do perfil dos pacientes internados em UTI sob VM é relevante, para nortear os padrões ventilatórios e a assistência ofertada.

Em relação ao Desmame do TOT, os participantes mantiveram a frequência de acertos insatisfatória de $47,7 \%$ tanto no pré como pós-teste. Para que se obtenha sucesso no desmame do paciente, estes devem ser submetidos ao teste de respiração espontânea, e para isso, faz-se necessário que os profissionais apresentem conhecimentos sobre estabilidade hemodinâmica, hemogasométrica e respiratória durante todo o período de avaliação ${ }^{(16)}$.

Neste estudo, foi possível observar o baixo nível de conhecimento sobre ventilação mecânica entre os acadêmicos da área da saúde, o que destaca a necessidade de maior abordagem sobre o tema durante a graduação com o intuito de capacitar esses indivíduos a prestar uma assistência qualificada e livre de danos tendo em vista que pacientes submetidos à ventilação mecânica estão expostos a procedimentos invasivos que podem acarretar complicações e óbitos.

\section{CONCLUSÃO}

O presente estudo demonstrou mudança significativa no nível de conhecimento sobre VM dos participantes envolvidos evidenciando que a implementação de cursos de capacitação representa a oportunidade dos acadêmicos atualizarem seus conhecimentos e se tornarem profissionais melhores na prática assistencial dos serviços de saúde, o que poderá favorecer a redução de infecções respiratórias associadas ao $\mathrm{VM}$ e a diminuição do tempo de internamento desses pacientes.

Os piores níveis de acertos foram relacionados à questões sobre Objetivo do Ventilador Mecânico; necessidade de gasometria, uso de músculos inspiratórios, os modos de operação [Modo PSV (Ventilação com Pressão de Suporte); Modo de Operação Combinada (SIMV); Modalidade Assistida Controlado a Pressão (PCV A/C)], Desmame do TOT Ciclagem por Pressão e Pausa Inspiratória. Após a intervenção educativa, houve aumento nos acertos das questões sobre Objetivo do Ventilador Mecânico, Gasometria, Modo de Operação Combinada (SIMV), Modo PSV (Ventilação com Pressão de Suporte), Ciclagem por Pressão e Pausa Inspiratória.

A educação continuada de estudantes e profissionais da saúde sobre VM é um meio para mudança de paradigma dos profissionais de saúde e corrobora para uma excelência no atendimento, além de reduzir os custos hospitalares. É de responsabilidade das instituições de ensino promover capacitações teóricas e práticas de manuseio do Ventilador Mecânico que resultem no ganho de conhecimentos e possibilitem um melhor cuidado ao paciente crítico.

O estudo apresentou limitações importantes quanto ao público-alvo, pois foram excluídos sete instrumentos de participantes que não responderam o pós-teste diante da impossibilidade de comparação dos resultados antes e depois da intervenção educativa. Este estudo poderia ser replicado em outros hospitais para se verificar a existência de variáveis de variáveis como tipo de curso e realização de estágios em campo hospitalar. 


\section{REFERÊNCIAS}

I. Torres-Castro C , Valle-Leal J , Martínez-Limón AJ , LastraJiménez Z , Delgado-Bojórquez LC . Pulmonary complications associated with mechanical ventilation in neonates. Boletín Médico Del Hospital Infantil de México (EnglishEdition) [Internet]. 2016 [acesso em 05 mar 2018]; 73(5):3 I824. Disponível em: https://www.sciencedirect.com/science/ article/pii/SI665 I |46 I6301046?via\%3Dihub.

2. Fernandez-Zamora MD, Gordillo-Brenes A, Banderas-Bravo E, Arboleda-Sánchez JA, Hinojosa-Pérez R, Aguilar-Alonso E, Herruzo-Aviles A, Curiel-Balsera E, Sánchez-Rodríguez A, Rivera-Fernández R. Prolonged mechanical ventilation as a predictor of mortality after cardiac surgery. Respiratory Care May [Internet]. 2018 [acesso em 08 jul 2018]; 63 (5) 550-557. Disponível em: http://rc.rcjournal. com/content/63/5/550.

3. Pontes LG, Menezes FG, Gonçalves P, Toniolo AR, Silva CV, Kawagoe JY, Santos CM, Castagna HMF, Martino MD, Corrêa L. Fatores de risco para mortalidade em traqueobronquite associada à ventilação mecânica: estudo caso-controle. Einstein (São Paulo) [Internet]. 2017 [acesso em 08 jul 2018]; I5( I ): 6I-64. Disponível em: http://www.scielo.br/scielo.php?script=sci_arttext\&pi$\mathrm{d}=\mathrm{S}|679-450820| 7000|0006|$ \&lng=en.

4. Silva J, Costa LMP, Figueiredo PO, Malta TS, Magalhães AO. Percepção do enfermeiro frente à segurança do paciente em uso de ventilação mecânica. TCC-ENFERMAGEM [Internet]. 2018 [acesso em I6 abr 20 I9]; I(I): I-I0. Disponível em: http://www.repositoriodigital.univag.com.br/index. php/enf/article/view/5I.

5. Cunha FA, Oliveira AFS. Complicações decorrentes da ventilação mecânica ao paciente de unidades de terapia intensiva (UTI) adulto. Health Research Journal [Internet]. 2018 [acesso em 09 mar 2019]; I(I): I38-16I. Disponível em: http://hrjbaq.ir/search.php?slc_lang=en\&sid=I .

6. Brasil. Agência Nacional de Vigilância Sanitária Medidas de Prevenção de Infecção Relacionada à Assistência à Saúde. Brasília:Anvisa, 2017.

7. Mansano FPN, Belei RA, Vinci LAS, Melo BLD, Cardoso LTQ, Garcia JCP, Carrilho CMDM, Ezaias GM, Grion CMC. Impacto de ação educativa na manutenção do decúbito elevado como medida preventiva de pneumonia associada à ventilação mecânica em Unidade de Terapia Intensiva. ABCS Health Sci [Internet]. 2017 [acesso em II abr 2019]; 42(I):2I-26 2. Disponível em: https://www.portalnepas.org. br/abcshs/article/view/945/757.

8. Yee J, Fuenning C, George R, Hejal R, Haines N, Dunn D, Gothard MD, Ahmed RA. Mechanical Ventilation Boot Camp: A Simulation-Based Pilot Study. Critical Care Research and Practice [Internet]. 2016 [acesso em I 3 abr 2019]; 2016(I): 1-7. Disponível em: https://www.hindawi.com/ journals/ccrp/2016/4670672/.

9. Costa JC, Machado JN, Costa J, Fortuna J, Gama J, Ro- drigues C. Ventilação Não Invasiva: Experiência de um Serviço de Medicina Interna. Medicina Interna [Internet]. 2018 [acesso em I3 abr 2019]; 25(I): 18-22. Disponível em: http://www.scielo.mec.pt/scielo.php?script=sci_arttext\&pid=S0872-67I IX2018000 I 00006\&lng=pt.

10. Jansson MM, Syrjälä HP, Ohtonen PP, Meriläinen $M H$, Kyngäs HA, Ala-Kokko TI. Randomized, controlled study of the effectiveness of simulation education: a 24-month follow-up study in a clinical setting. Am J InfectControl [Internet]. 2016 [acesso em 20 abr 2019]; 44(4): 38793. Disponível em: https://www.ncbi.nlm.nih.gov/pubmed/26708025.

II. Pinto JMA, Saracini KC, Lima LCA, Souza LP, Lima MG, Algeri EDBO. Gasometria arterial: aplicações e implicações para a enfermagem. Revista Amazônia Science \& Health [Internet]. 2017 [ acesso em 21 abr 20I9]; 5(2): 33-9. Disponível em: http://ojs.unirg.edu.br/index.php/2/article/ view/I I I7/pdf.

12. Padma K, Priya A, Subhashini N, Indira S. A study to assess knowledge about $A B G$ analysis and interpretation of $A B G$ results among nurses from the $\mathrm{NMCH}$ team, Nelore. International Journal of Applied Research [Internet]. 2017 [acesso em 23 abr 2019]; 3(I): 06-08. Disponível em: http:// www.allresearchjournal.com/archives/20 I 7/vol3issue I/ PartA/2-12-122-442.pdf.

13. Melo EM, Oliveira AKC, Lima VF, Garces TS, Araújo SS, Silveira FMM, Ferreira AMM. Avaliação dos parâmetros ventilatórios em pacientes de unidade de terapia intensiva. Rev enferm UFPE [Internet]. 2017 [acesso em $23 \mathrm{abr}$ 20 I9]; I I (3): I 375-80. Disponível em: I0.5205/reuol. I02639I568-I-RV.I I03sup20I708.

14. Loss SH, Oliveira RP, Maccari JG, Savi A, Boniatti MM, Hetzel MP, Dallegrave DM, Balzano PC, Oliveira ES, Höher JA, Torelly AP, Teixeira C. A realidade dos pacientes que necessitam de ventilação mecânica prolongada: um estudo multicêntrico. Rev. bras. ter. intensiva [Internet]. 2015 [acesso em 0I mai 2019]; 27(I):26-35. Disponível em: http:// www.scielo.br/scielo.php?script=sci_arttext\&pi$d=S 0103-507 \times 2015000100026 \&$ Ing=en.

15. Barbas CSV, Ísola AM, Farias AMC. Diretrizes Brasileiras de Ventilação Mecânica (20I3). la.ed. São Paulo: AMIB; 20I3.p.19-22.

16. Struck MF, Fakler, JKM, Bernhard M, Busch T, Stumpp P, Hempel G, Beilicke A, Stehr SN, Josten C, Wrigge H. Mechanical complications and outcomes following invasive emergency procedures in severely injured trauma patients. Sci Rep [Internet]. 2018 [acesso em 01 mai 2019]; 8, 3976 (20/8). Disponível em: https://doi.org//0.1038/ s41598-018-22457-9.

Recebido: 2020-04-23

Aceito: 2020- I I-04 